\title{
Determination of Cochlear Duct Length With 3D Versus Two-dimensional Methods: A Retrospective Clinical Study of Imaging by Computed Tomography and Cone Beam Computed Tomography
}

\author{
STEPHAN WALDECK ${ }^{1,2}$, CHRISTIAN VON FALCK $^{3}$, RENE CHAPOT ${ }^{4}$, \\ MARC BROCKMANN ${ }^{2}$ and DANIEL OVERHOFF ${ }^{1,5}$ \\ ${ }^{1}$ Department of Diagnostic and Interventional Radiology and Neuroradiology, \\ Bundeswehr Central Hospital Koblenz, Koblenz, Germany; \\ ${ }^{2}$ Institute of Neuroradiology, University Medical Centre Johannes Gutenberg University Mainz, Mainz, Germany; \\ ${ }^{3}$ Institute of Diagnostic and Interventional Radiology, Hannover Medical School, Hanover, Germany; \\ ${ }^{4}$ Department of Neuroradiology, Alfried Krupp Krankenhaus, Essen, Germany; \\ ${ }^{5}$ Department of Radiology and Nuclear Medicine, University Medical Centre Mannheim, \\ Medical Faculty Mannheim, Heidelberg University, Mannheim, Germany
}

\begin{abstract}
Background: The aim of this study was to compare three different methods for measurement of cochlear duct length $(C D L)$ in the clinical setting for two different imaging modalities, namely computed tomography $(C T)$ and cone-beam computed tomography (CBCT). Patients and Methods: One hundred temporal bone data sets (CT: $n=50$; $C B C T: n=50)$ of non-malformed cochleae were retrospectively analyzed using three different $C D L$ estimation techniques: $3 D$ curved multiplanar reconstruction (cMPR), $2 D$ cMPR and the A-value formula. Results: The data sets belonged to 60 patients (34 males, 26 females; mean age $=50.28 \pm 18.58$ years). For both imaging modalities, application of the $3 D$ cMPR estimation technique led to significantly greater mean $C D L$ values than the two-dimensional methods $(p<0.0083)$. The CDL measurements viewed in CT imaging software were significantly shorter than the corresponding CBCT measurements $(p<0.05)$. Using a linear mixed model, differences in CDL by sex $(p=0.796)$, age $(p=0.377)$ and side of ear $(p=0.690)$ were not significant. Conclusion: The $3 D$
\end{abstract}

This article is freely accessible online.

Correspondence to: Stephan Waldeck, MD, Department of Diagnostic and Interventional Radiology and Neuroradiology, Bundeswehr Central Hospital Koblenz, Rübenacherstrassse 170, 56072 Koblenz, Germany. Tel: +49 26128128000, Fax: +49 26128128009, e-mail: dr.waldeck@icloud.com

Key Words: Cochlear duct length, 3D curved multiplanar reconstruction, computed tomography, cone beam computed tomography, A-value.
cMPR technique was found to provide the most accurate in vivo CDL measurement in non-malformed cochlea in both CT and CBCT imaging compared to $2 D$ methods. The study results also suggest that the higher spatial resolution in $C B C T$ imaging results in more precise CDL determination than in $C T$.

Cochlear implant surgery requires accurate knowledge of the cochlear duct length (CDL) as the basis in treatment planning. The literature reveals varying percentages of interindividual differences between CDL according to gender $(1,2)$, ethnicity (1-3) and imaging modality (4-6) used to determine CDL. Over-insertion of the electrode during cochlea implant surgery may result in trauma to the cochlear structure and loss of residual hearing $(7,8)$. However, electrode under-insertion or insufficient electrode length can lead to inadequate coverage of the available frequency range and unsatisfactory hearing with an implant $(9,10)$. In contrast, an optimal electrode length that matches patientspecific cochlear anatomy and corresponds to the tonotopic locations greatly enhances speech recognition $(11,12)$.

To date, only few studies have compared three-dimensional (3D) with two-dimensional (2D) CDL estimation methods. In older studies, 3D reconstruction proved superior to $2 \mathrm{D}$ CDL estimation techniques due to reduced influence of the cuttingangle effect (13-16). Würfel et al. were the first to use a 3D curved multiplanar reconstruction (cMPR) model in conebeam computed tomography (CBCT) scans to accurately represent the cochlea (5). Meng et al. followed with a study of 3D cMPR for CDL determination in computed tomography (CT) scans (17). 


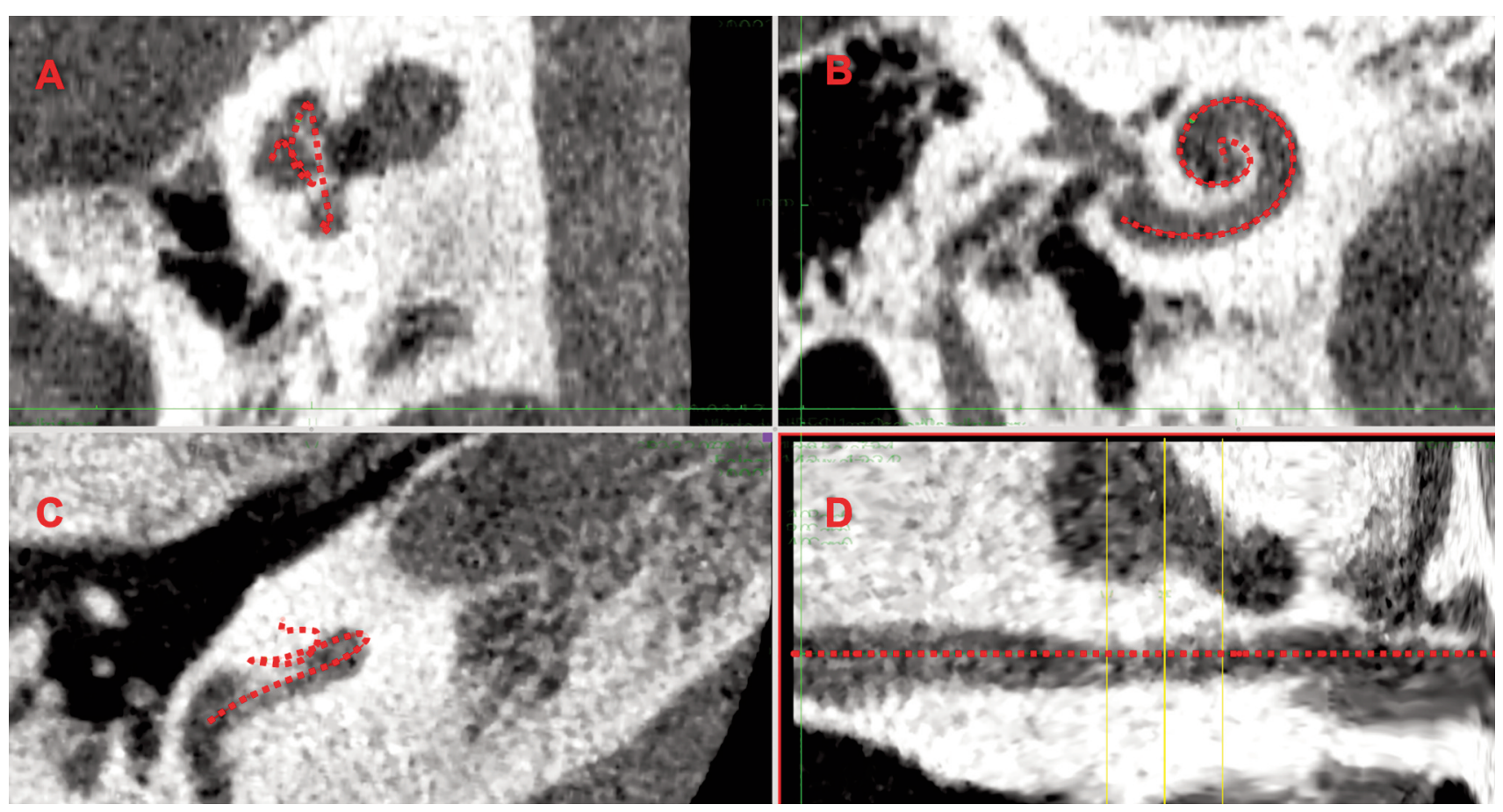

Figure 1. Method 1:3D curved multiplanar reconstruction (cMPR). 3D cMPR of the cochlea, indicated by the red line in the paracoronal plane $(A)$, transverse plane $(B)$, unformatted plane of the cochlea $(C)$ and $c M P R(D)$.

However, we were unable to identify any studies directly comparing 3D and 2D CDL estimation methods using both CT and CBCT imaging in a clinical setting. This retrospective study therefore had multiple aims, namely i) to compare three different methods of CDL measurement, ii) to analyze CDL measurements for the imaging modalities $\mathrm{CT}$ and $\mathrm{CBCT}$, and iii) to determine possible effects of sex and age on CDL, as well as detect intra-individual differences in CDL between the left and right ear.

\section{Patients and Methods}

Patients and datasets. This retrospective study was approved by the local Ethics Committee (approval number: 2021-15895). One hundred radiological data sets (Bundeswehr Central Hospital Koblenz) from 60 patients (34 males, 26 females) were retrieved for this study. The patients had undergone an anatomical evaluation of their temporal bones with either $\mathrm{CT}$ ( $\mathrm{n}=50$ data sets) or $\mathrm{CBCT}$ ( $\mathrm{n}=50$ data sets) as part of a clinical visit for differential diagnosis of hearing loss between October 2012 and November 2016. We excluded data sets when anomalies were present in either the cochlea or the temporal bone.

Cochlea length measurement. For all included data sets, CDL was evaluated with three separate methods:

Method 1: 3D cMPR was constructed from manually defined points along the lateral wall of the bony cochlea (Figure 1), using the technique described by Würfel et al. (5).

Method 2: CDL estimation based on the diameter of the basal turn (A-value) (Figure 2) by applying the following equation for complete CDL: CDL (measured at the organ of Corti) $=4.16 \mathrm{~A}-3.98$. The method is presented in detail by Alexiades et al. (18).

Method 3: This CDL estimation method relied on measurements performed on 2D cMPR images constructed at the level of each of the three turns (basal, middle and apical). Examination of the cochlea was performed for the transverse plane, with levels aligned according to the center of the osseous cochlear structure. After angle correction, the individual turn lengths were added to derive the total CDL (Figure 3).

Statistical analysis. Initially, an analysis of variance test was used to test for significant differences between the three CDL estimation methods within each of the two imaging modalities. Post-hoc testing for differences between the CDL estimation methods was performed with either a paired Student's $t$-test or Wilcoxon signed-rank test. The significance level was set at $p<0.05$ and the Bonferroni-corrected $p$-value of 0.0083 was used to adjust for multiple testing.

A linear mixed model was used to analyze possible differences in length measurement between the examination modalities CBCT and CT. The dependent variable was cochlear length measurement. Fixed effects were defined as age, examination modality, gender, measurement method and side; the individual patient was defined as a random effect.

\section{Results}

The mean age of the study population was $50.28 \pm 18.58$ years. The baseline characteristics of the study population are given in Table I. 


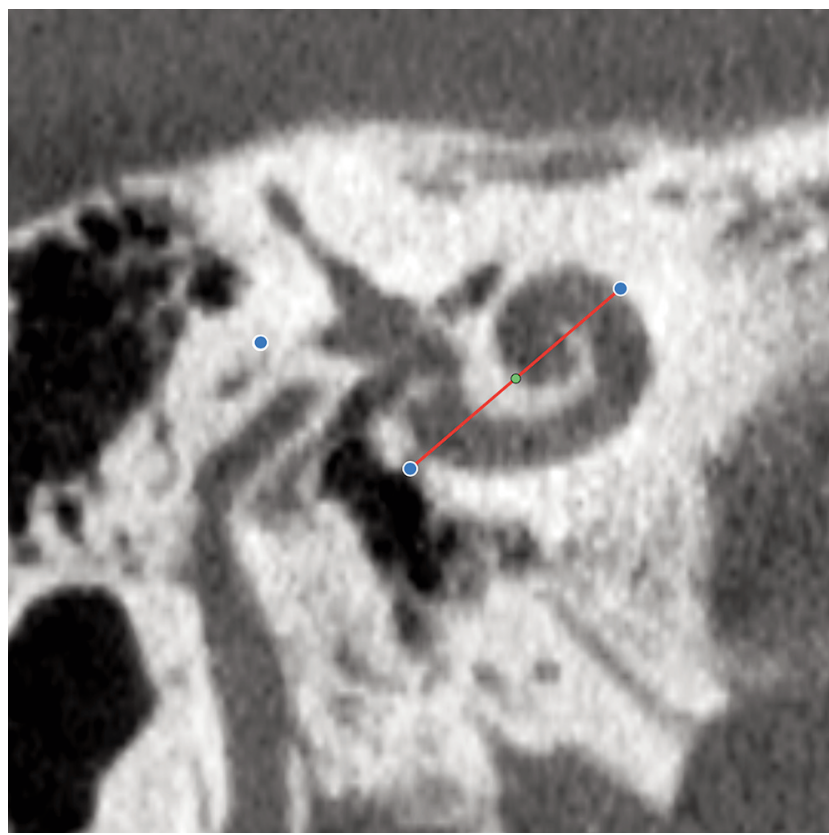

Figure 2. Method 2 - cochlear duct length estimation via A-value determination, indicated by the red line.

Analysis of variance detected significant differences in CDL between the three estimation methods applied for both CT $(p<0.001)$ and CBCT $(p<0.001)$ imaging modalities.

Comparison of CDL estimation method by CT imaging. The mean CDL determined by application of 3D cMPR measurement technique was $31.964 \pm 1.668 \mathrm{~mm}$ and that using 2D cMPR was $31.056 \pm 1.825 \mathrm{~mm}$ for the CT images examined.

When using the equation (A-value) for CDL estimation, the mean length was $31.151 \pm 1.631 \mathrm{~mm}$. The CDL was significantly longer (mean difference $\Delta=0.908 \mathrm{~mm} ; p<0.001$ ) when measured using the $3 \mathrm{D}$ cMPR method versus $2 \mathrm{D} \mathrm{cMPR}$. CDL estimation with the $3 \mathrm{D} \mathrm{cMPR}$ method resulted in significantly greater (mean difference $\Delta=0.812 \mathrm{~mm} ; p<0.001$ ) $\mathrm{CDL}$ values than the A-value equation. The comparison between the results of the $2 \mathrm{D}$ cMPR measurement and those of the A-value equation revealed a slighter greater CDL estimation when using the latter method. This difference of $0.096 \mathrm{~mm}$ did not reach statistical significance $(p=0.604)$.

Comparison of CDL estimation methods by CBCT imaging. The average CDL was $33.146 \pm 1.947 \mathrm{~mm}$ when using the $3 \mathrm{D}$ cMPR technique and CBCT imaging. The 2D cMPR method led to a mean CDL of $31.844 \pm 2.108 \mathrm{~mm}$ and the A-value equation generated a mean CDL of $32.625 \pm 1.667 \mathrm{~mm}$ for the CBCT imaging modality.

Comparison between the $3 \mathrm{D}$ and $2 \mathrm{D}$ cMPR methods showed significantly greater CDL (mean difference $\Delta=1.302$
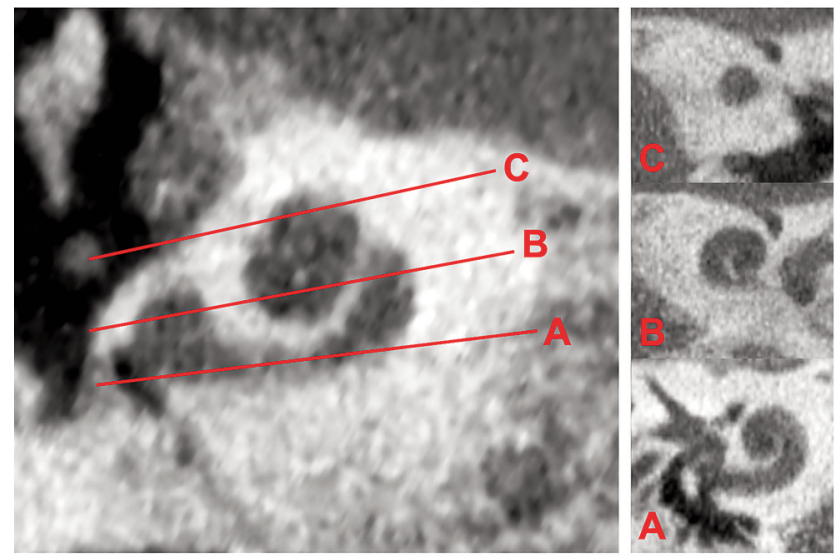

Figure 3. Method $3-2 D$ curved multiplanar reconstruction (cMPR). Application of $2 D$ cMPR method with level alignment in the transverse plane, indicated by red lines $(A, B$, and $C)$, followed by measurements of the basal turn (A), middle turn $(B)$ and apical turn $(C)$.

$\mathrm{mm} ; p<0.001)$ when using the 3D cMPR technique. The CDL estimation with the 3D cMPR method led to significantly greater CDL values (mean difference $\Delta=0.521$ $\mathrm{mm} ; p<0.007)$ than using the A-value equation. In CBCT imaging, use of the A-value equation led to greater CDL values than the $2 \mathrm{D}$ cMPR technique. This difference of 0.781 mm was statistically significant $(p=0.009)$.

Results of the linear mixed model. When comparing the imaging modalities, the linear mixed model detected a significant difference $(p=0.005)$ in mean CDL estimations. The CDL measurements viewed in CT imaging software were $1.222 \mathrm{~mm}$ shorter $[95 \%$ confidence interval $(\mathrm{CI})=-2.064--0.380 \mathrm{~mm}$ ] than the corresponding CBCT measurements. The CDL for males was on average 0.110 (95\% CI $=-0.959--0.739) \mathrm{mm}$ shorter than that for females but this difference was not significant $(p=0.796)$. Even though the mean CDL for the right ear was 0.051 (95\% CI $=-0.200-0.302) \mathrm{mm}$ longer than that of the left ear, the difference was not significant $(p=0.690)$. Regarding age, the model showed an average CDL increase of $0.010(95 \% \mathrm{CI}=-0.125-0.033) \mathrm{mm}$ per year (Figure 4), which did not reach statistical significance $(p=0.377)$.

Finally, a linear mixed model incorporating the effects of measurement method, age, sex and ear was used to calculate the estimated mean for the three CDL estimation techniques to allow comparison with the literature (Table II).

\section{Discussion}

To the best of our knowledge, this is the first study that directly compared 3D and 2D CDL estimation methods using 
Table I. Baseline characteristics of the study population.

\begin{tabular}{lccc}
\hline & Total & CT & CBCT \\
\hline $\mathrm{n}$ & 60 & 25 & 35 \\
$\begin{array}{l}\text { Gender, n (\%) } \\
\quad \text { Male }\end{array}$ & $34(56.7 \%)$ & $12(48 \%)$ & $22(62.9 \%)$ \\
$\quad$ Female & $26(43.3 \%)$ & $13(52 \%)$ & $13(37.1 \%)$ \\
Age, years & & & \\
$\quad$ Mean \pm SD & $50.28 \pm 18.58$ & $50.80 \pm 21.14$ & $49.91 \pm 16.82$ \\
$\quad$ Range & $5-87$ & $5-87$ & $8-77$ \\
\hline
\end{tabular}

CBCT: Cone-beam computed tomography; CT: computed tomography; SD: standard deviation.

Table II. Results of linear mixed model for estimated mean cochlea duct length according to calculation method and imaging modality.

\begin{tabular}{lcccc}
\hline $\begin{array}{l}\text { Imaging } \\
\text { modality }\end{array}$ & Method & $\begin{array}{c}\text { Mean } \\
(\mathrm{mm})\end{array}$ & $\begin{array}{c}\text { Standard } \\
\text { error (mm) }\end{array}$ & 95\% CI \\
\hline CT $^{\mathrm{a}}$ & 3D cMPR & 31.974 & 0.325 & $31.307-32.642$ \\
& A-value & 31.162 & 0.325 & $30.495-31.829$ \\
& 2D cMPR & 31.066 & 0.325 & $30.399-31.734$ \\
$\mathrm{CBCT}^{\mathrm{b}}$ & 3D cMPR & 33.275 & 0.318 & $32.636-33915$ \\
& A-value & 32.754 & 0.318 & $32.115-33395$ \\
& 2D cMPR & 31.973 & 0.318 & $31.334-32.913$
\end{tabular}

CBCT: Cone-beam computed tomography; CI: confidence intervaI; cMPR: curved multiplanar reconstruction; CT: computed tomography. aModelled on mean age of 50.80 years; bmodelled on mean age of 52.46 years.

both CT and CBCT imaging in a clinical setting. The 3D cMPR method for CDL measurement proved more accurate than both $2 \mathrm{D}$ estimation techniques. These findings are in line with the literature (19) and highly relevant from a clinical perspective. Rivas et al. found that selection of a cochlear implant electrode array was influenced by the choice of algorithm used in CDL determination, with mean absolute differences of $1.15 \mathrm{~mm}$ between CDL estimations (20). Timm et al. suggested that CDL estimations are not suitable for clinical use, where accuracy is paramount in each individual patient case as over- or underestimation of CDL was found in $33 \%$ of the analyzed cochleae (21). Thus, it follows that the degree of variability involved in CDL determination may lead to completely different choices of cochlear implant electrode array, depending on the manufacturer-specific threshold values for the array type.

Most of the current methods of CDL estimation in use today rely on calculations from parameters measured in the basal turn $(18,22)$. However, due to the complex anatomy of the cochlea, these formulae remain approximations, even though many authors have attempted to improve the accuracy of these equations and models by adding additional parameters such as basal turn width (22) and spiral coefficients $(23,24)$. In a recent analysis of methods and results, Koch et al. detailed the many pitfalls associated with 2D CDL estimations and suggested that the 3D cMPR method significantly reduces the time needed to perform reconstruction compared to the use of histological sections (19). Another study found that manual CDL determination by cMPR required an average of $9.3 \pm 0.72$ minutes per cochlea, while a semi-automated approach with a new otological planning software program needed only $5.9 \pm 0.69$ minutes (25).

In this study, the estimated means for 3D cMPR were $31.964 \pm 1.668 \mathrm{~mm}$ with CT and $33.146 \pm 1.947 \mathrm{~mm}$ with CBCT. Although measured along the lateral wall, these CDL correspond more with CDL measurements taken at the level of the organ Corti $(16,26)$. This difference was attributed partly to the study population itself, whose A-values show that the included patients belonged to the middle group with smaller cochleae as defined in a study by Escude et al. (24). In addition, the strict intraluminal measurement performed in our study may have led to systematically shorter CDL estimations. Finally, Koch et al. note there is a considerable amount of variation surrounding CDL in studies utilizing CT scans due to inter-observer variability when placing the curve, the lack of standardized guidelines as to where measurements are to be taken, and differences between observers in apex selection (19). Standardization of CDL measurement protocols may improve both accuracy and consistency in CDL determination.

In this study, CBCT provided more accurate CDL estimations than $\mathrm{CT}$ scans, irrespective of the calculation method used. These findings are in accordance with the literature in CBCT imaging in cadaveric studies, in which CBCT displayed good agreement for cochlear metrics (27, 28). There is, however, considerable variation between CBCT and CT scanners and their respective viewing software programs, which might considerably influence CDL determination (29). 3D cMPR relies on accurate detection of structures in radiological imaging, a factor that is positively influenced by the higher spatial resolution available in CBCT. The lower radiation dosages compared to conventional CT make CBCT imaging an optimal choice for CDL estimation.

This study is mainly limited by the small sample size and single-center design. Future research with thinner scanner slices is needed to confirm these findings.

\section{Conclusion}

The 3D cMPR technique provides the most accurate in vivo measurement of $\mathrm{CDL}$ for both $\mathrm{CT}$ and $\mathrm{CBCT}$ imaging compared to $2 \mathrm{D}$ methods in non-malformed cochlea. This should be considered in treatment planning, seeing how variations in CDL determination may lead to a completely 


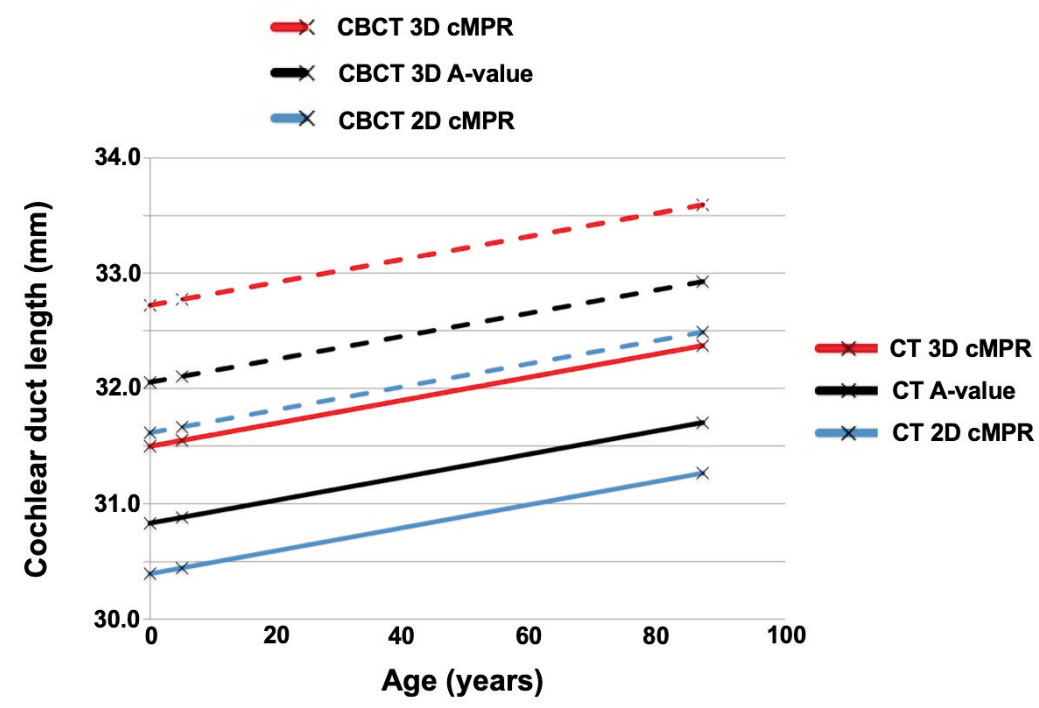

Figure 4. Linear mixed model. Linear mixed model for effect of age on cochlear duct length for the two imaging modalities and three different estimation methods. CBCT: Cone-beam computed tomography; cMPR: curved multiplanar reconstruction; CT: computed tomography.

different choice of cochlear implant electrode array, depending on the manufacturer-specific threshold values for the array type.

The study results also suggest that the higher spatial resolution in $\mathrm{CBCT}$ imaging results in more precise $\mathrm{CDL}$ determination than using plain CT measurements.

\section{Conflicts of Interest}

None.

\section{Authors' Contributions}

All Authors conceived and designed the analysis, collected the data, contributed data, performed the analysis, and wrote the article.

\section{References}

1 Thong JF, Low D, Tham A, Liew C, Tan TY and Yuen HW: Cochlear duct length-one size fits all? Am J Otolaryngol 38(2): 218-221, 2017. PMID: 28139318. DOI: 10.1016/j.amjoto. 2017.01.015

2 Alanazi A and Alzhrani F: Comparison of cochlear duct length between the Saudi and non-Saudi populations. Ann Saudi Med 38(2): 125-129, 2018. PMID: 29620546. DOI: 10.5144/02564947.2018.125

3 Kumar JU and Kavitha Y: Application of curved MPR algorithm to high resolution 3 dimensional T2 weighted CISS images for virtual uncoiling of membranous cochlea as an aid for cochlear morphometry. J Clin Diagn Res 11(2): TC12-TC14, 2017. PMID: 28384958. DOI: $10.7860 / J C D R / 2017 / 23206.9456$

4 Connor SE, Bell DJ, O'Gorman R and Fitzgerald-O'Connor A: $\mathrm{CT}$ and MR imaging cochlear distance measurements may predict cochlear implant length required for a 360 degrees insertion. AJNR Am J Neuroradiol 30(7): 1425-1430, 2009. PMID: 19386728. DOI: 10.3174/ajnr.A1571

5 Würfel W, Lanfermann H, Lenarz T and Majdani O: Cochlear length determination using Cone Beam Computed Tomography in a clinical setting. Hear Res 316: 65-72, 2014. PMID: 25124151. DOI: $10.1016 /$ j.heares.2014.07.013

6 Singh A, Kumar R, Manchanda S, Bhalla AS, Sagar P and Irugu DVK: Radiographic measurement of cochlear duct length in an Indian Cadaveric population - Importance of custom fit cochlear implant electrodes. Int Arch Otorhinolaryngol 24(4): e492-e495, 2020. PMID: 33101517. DOI: 10.1055/s-0040-1701272

7 Carlson ML, Driscoll CL, Gifford RH, Service GJ, Tombers NM, Hughes-Borst BJ, Neff BA and Beatty CW: Implications of minimizing trauma during conventional cochlear implantation. Otol Neurotol 32(6): 962-968, 2011. PMID: 21659922. DOI: 10.1097/MAO.0b013e3182204526

8 Nordfalk KF, Rasmussen K, Hopp E, Bunne M, Silvola JT and Jablonski GE: Insertion depth in cochlear implantation and outcome in residual hearing and vestibular function. Ear Hear 37(2): e129-e137, 2016. PMID: 26524566. DOI: 10.1097/ AUD.0000000000000241

9 O'Connell BP, Cakir A, Hunter JB, Francis DO, Noble JH, Labadie RF, Zuniga G, Dawant BM, Rivas A and Wanna GB: Electrode location and angular insertion depth are predictors of audiologic outcomes in cochlear implantation. Otol Neurotol 37(8): 1016-1023, 2016. PMID: 27348391. DOI: 10.1097/ MAO.0000000000001125

10 Lenarz T, Timm ME, Salcher R and Büchner A: Individual hearing preservation cochlear implantation using the concept of partial insertion. Otol Neurotol 40(3): e326-e335, 2019. PMID: 30741914. DOI: 10.1097/MAO.0000000000002127

11 Buchman CA, Dillon MT, King ER, Adunka MC, Adunka OF and Pillsbury HC: Influence of cochlear implant insertion depth on performance: a prospective randomized trial. Otol Neurotol 
35(10): 1773-1779, 2014. PMID: 25122601. DOI: 10.1097/ MAO.0000000000000541

12 Canfarotta MW, Dillon MT, Buss E, Pillsbury HC, Brown KD and O'Connell BP: Frequency-to-place mismatch: Characterizing variability and the influence on speech perception outcomes in cochlear implant recipients. Ear Hear 41(5): 13491361, 2020. PMID: 32205726. DOI: 10.1097/AUD. 0000000000000864

13 Takagi A and Sando I: Computer-aided three-dimensional reconstruction: a method of measuring temporal bone structures including the length of the cochlea. Ann Otol Rhinol Laryngol 98(7 Pt 1): 515-522, 1989. PMID: 2751211. DOI: 10.1177/ 000348948909800705

14 Green JD Jr, Marion MS, Erickson BJ, Robb RA and Hinojosa $\mathrm{R}$ : Three-dimensional reconstruction of the temporal bone. Laryngoscope 100(1): 1-4, 1990. PMID: 2293695. DOI: 10.1288/00005537-199001000-00001

15 Sato H, Sando I and Takahashi H: Sexual dimorphism and development of the human cochlea. Computer 3-D measurement. Acta Otolaryngol 111(6): 1037-1040, 1991. PMID: 1763623. DOI: $10.3109 / 00016489109100753$

16 Kawano A, Seldon HL and Clark GM: Computer-aided threedimensional reconstruction in human cochlear maps: measurement of the lengths of organ of Corti, outer wall, inner wall, and Rosenthal's canal. Ann Otol Rhinol Laryngol 105(9): 701-709, 1996. PMID: 8800056. DOI: 10.1177/0003489496 10500906

17 Meng J, Li S, Zhang F, Li Q and Qin Z: Cochlear size and shape variability and implications in cochlear implantation surgery. Otol Neurotol 37(9): 1307-1313, 2016. PMID: 27579839. DOI: 10.1097/MAO.0000000000001189

18 Alexiades G, Dhanasingh A and Jolly C: Method to estimate the complete and two-turn cochlear duct length. Otol Neurotol 36(5): 904-907, 2015. PMID: 25299827. DOI: 10.1097/ MAO.0000000000000620

19 Koch RW, Ladak HM, Elfarnawany $M$ and Agrawal SK: Measuring Cochlear Duct Length - a historical analysis of methods and results. J Otolaryngol Head Neck Surg 46(1): 19, 2017. PMID: 28270200. DOI: 10.1186/s40463-017-0194-2

20 Rivas A, Cakir A, Hunter JB, Labadie RF, Zuniga MG, Wanna GB, Dawant BM and Noble JH: Automatic cochlear duct length estimation for selection of cochlear implant electrode arrays. Otol Neurotol 38(3): 339-346, 2017. PMID: 28146009. DOI: 10.1097/MAO.0000000000001329

21 Timm ME, Majdani O, Weller T, Windeler M, Lenarz T, Büchner A and Salcher RB: Patient specific selection of lateral wall cochlear implant electrodes based on anatomical indication ranges. PLoS One 13(10): e0206435, 2018. PMID: 30365565. DOI: 10.1371 /journal.pone. 0206435
22 Khurayzi T, Almuhawas F and Sanosi A: Direct measurement of cochlear parameters for automatic calculation of the cochlear duct length. Ann Saudi Med 40(3): 212-218, 2020. PMID: 32493102. DOI: $10.5144 / 0256-4947.2020 .218$

23 Ketten DR, Skinner MW, Wang G, Vannier MW, Gates GA and Neely JG: In vivo measures of cochlear length and insertion depth of nucleus cochlear implant electrode arrays. Ann Otol Rhinol Laryngol Suppl 175: 1-16, 1998. PMID: 9826942.

24 Escudé B, James C, Deguine O, Cochard N, Eter E and Fraysse $\mathrm{B}$ : The size of the cochlea and predictions of insertion depth angles for cochlear implant electrodes. Audiol Neurootol 11 Suppl 1: 27-33, 2006. PMID: 17063008. DOI: 10.1159/ 000095611

25 Chen Y, Chen J, Tan H, Jiang M, Wu Y, Zhang Z, Li Y, Jia H and $\mathrm{Wu} \mathrm{H}$ : Cochlear Duct length calculation: Comparison between using otoplan and curved multiplanar reconstruction in nonmalformed cochlea. Otol Neurotol 42(7): e875-e880, 2021. PMID: 33710146. DOI: 10.1097/MAO.0000000000003119

26 Stakhovskaya O, Sridhar D, Bonham BH and Leake PA: Frequency map for the human cochlear spiral ganglion: implications for cochlear implants. J Assoc Res Otolaryngol 8(2): 220-233, 2007. PMID: 17318276. DOI: 10.1007/s10162007-0076-9

27 Guyader E, Savéan J, Clodic C, Letellier P, Meriot P and Marianowski R: Three-dimensional reconstruction of the temporal bone: Comparison of in situ, CT, and CBCT measurements. Eur Ann Otorhinolaryngol Head Neck Dis 135(6): 393-398, 2018. PMID: 30220575. DOI: 10.1016/ j.anorl.2018.08.013

28 Nateghifard K, Low D, Awofala L, Srikanthan D, Kuthubutheen J, Daly M, Chan H, Irish J, Chen J, Lin V and Le TN: Cone beam $\mathrm{CT}$ for perioperative imaging in hearing preservation Cochlear implantation - a human cadaveric study. J Otolaryngol Head Neck Surg 48(1): 65, 2019. PMID: 31753027. DOI: 10.1186/s40463-019-0388-x

29 Liang X, Jacobs R, Hassan B, Li L, Pauwels R, Corpas L, Souza PC, Martens W, Shahbazian M, Alonso A and Lambrichts I: A comparative evaluation of Cone Beam Computed Tomography (CBCT) and Multi-Slice CT (MSCT) Part I. On subjective image quality. Eur J Radiol 75(2): 265-269, 2010. PMID: 19410409. DOI: $10.1016 /$ j.ejrad.2009.03.042 